\title{
AN APPROXIMATE POSSIBILISTIC GRAPHICAL MODEL FOR COMPUTING OPTIMISTIC QUALITATIVE DECISION
}

\author{
BOUTOUHAMI Khaoula and KHELLAF Faiza \\ Recherche en Informatique Intelligente et Mathématiques Appliquées. \\ Université des Sciences et de la Technologie Houari Boumediene. \\ Algiers, Algeria \\ boutouhami_khaoula@yahoo.fr, hanedfaiza@yahoo.fr
}

\begin{abstract}
Min-based qualitative possibilistic networks are one of the effective tools for a compact representation of decision problems under uncertainty. The exact approaches for computing decision based on possibilistic networks are limited by the size of the possibility distributions. Generally, these approaches are based on possibilistic propagation algorithms. An important step in the computation of the decision is the transformation of the DAG into a secondary structure, known as the junction trees. This transformation is known to be costly and represents a difficult problem. We propose in this paper a new approximate approach for the computation of decision under uncertainty within possibilistic networks. The computing of the optimal optimistic decision no longer goes through the junction tree construction step. Instead, it is performed by calculating the degree of normalization in the moral graph resulting from the merging of the possibilistic network codifying knowledge of the agent and that codifying its preferences.
\end{abstract}

\section{KEYWORDS}

Possibilistic decision theory, Min-based possibilistic networks, Moral graph, optimistic criteria.

\section{INTRODUCTION}

Decision making under uncertainty plays an important role in Artificial Intelligence (AI) applications. Several decision making tools have been developed to assist decision makers in their tasks: simulation techniques, dynamic programming, logical decision models and graphical decision models. This paper focuses on graphical decision models which provide efficient decision tools by allowing a compact representation of decision problems under uncertainty [14]. A decision problem is a choice between a list of possible alternatives taking into account the knowledge of an agent (knowledge is sometimes tainted with uncertainties) as well as his/her preferences. The results of his/her decision are expressed by a set of utilities. The qualitative possibilistic decision model allows a progressive expression of preferences as well as knowledge of the decision-maker. This model offers two qualitative criteria of utilities for the approach of decision under uncertainty: the pessimistic decision criterion and the optimistic decision criterion. Interest in the issue of the calculation of qualitative decision continues to grow and many approaches and models have been proposed [7][12].

Dhinaharan Nagamalai et al. (Eds) : CCSEA, DKMP, AIFU, SEA - 2015

pp. 183-196, 2015. (C) CS \& IT-CSCP 2015

DOI : $10.5121 /$ csit. 2015.50216 
In addition to the calculation of decision, the aim of this method is to improve other methods and overcome their limits as regards the presentation form, the calculation time as well as the ease of understanding. In our work we focus on graphical decision models that provide effective tools for decision problems under uncertainty using a compact representation. Several evaluation methods have been proposed to select the optimal decision. Among these methods, there is an exact approach based on possibilistic networks spread. This approach requires a transformation of an original graph into a secondary structure called the junction tree [11] which is used then in various calculations. In this work, our goal is to propose a new approximate approach to compute the optimal optimistic decision. Our approach is based on the moral graph associated with the result of merging the networks representing the agent's beliefs and preferences. This approach has a polynomial complexity [1]. Indeed, it avoids the transformation of the initial graph into a junction tree which is known to be an intractable problem (NP-hard). Using the approximate approach provides very close answers to the exact marginal distributions [1].

The reminder of the paper is organized as follows. The next section briefly recalls the fundamental concepts of possibility theory and min-based possibilistic networks. The main results of merging min-based possibilistic networks are also briefly presented in this section. Section 3 describes the new approach and its use in calculating the optimal optimistic decision and section 4 concludes the paper.

\section{BACKGROUND}

\subsection{Basic concepts of possibility theory}

This section gives a brief refresher on possibility theory which is issued from fuzzy sets theory [16], [13] and represents a main approach in dealing with uncertainty. Let $\mathcal{V}=\left\{\mathrm{A}_{1}, \mathrm{~A}_{2}, \ldots, \mathrm{A}_{\mathrm{n}}\right\}$ be a set of variables. We denote by $D_{A}=\left\{a_{1}, \ldots, a_{n}\right\}$ the domain associated with the variable $A$ $a_{i}$ denotes any instance of $A$. The universe of discourse is denoted by $\Omega=\times \times_{A_{i} \in V} D_{A}$, which is the Cartesian product of all variable domains in $\mathcal{V}$. Each element $\omega \in \Omega$ is called an interpretation which represents a possible state of the world. It is denoted by $\omega=\left(a_{1}, \ldots, a_{n}\right)$ or $\omega=\left(a_{1} \wedge a, \ldots \wedge a_{n}\right)$. Where $\left\{a_{\mathrm{i}} \mid 1 \leq \mathrm{i} \leq \mathrm{n}\right\}$ are the instances of the variable $\mathrm{A}_{\mathrm{i}} \cdot \phi, \psi$ denote propositional formulas (corresponding to events, i.e., subsets of $\Omega$ ) constituted from the variables in $\mathcal{V}$.

\subsubsection{Possibility distribution}

The basic element in possibility theory is the notion of possibility distribution $\pi$ which is a mapping from $\Omega$ to the scale $[0,1]$. This distribution encodes available knowledge on real world: $\pi(\omega)=1$ means that $\omega$ is completely possible and $\pi(\omega)=0$ means that it is impossible to $\omega$ to be the real world. A possibility distribution $\pi$ is said to be $\alpha-$ normlized, if its normalization degree $h(\pi)$ is equal to $\alpha$, namely:

$$
h(\pi)=\max \pi(\omega)=\alpha
$$

If $\alpha=1$, then $\phi$ is said to be normalized.

Given a possibility distribution $\phi$ on the universe of discourse $\Omega$, two dual measures are defined for each event $\phi \subseteq \Omega$ :

- Possibility measure: this measure evaluates to what extent $\phi$ is consistent with our knowledge. It is given by: 


$$
\Pi(\phi)=\{\pi(\omega): \omega \in \phi\}
$$

- Necessity measure: it is the dual of the possibility measure. The necessity measure evaluates at which level $\phi$ is certainly implied by our knowledge. It is given by:

$$
\mathrm{N}(\phi)=1-\Pi(\bar{\phi})
$$

\subsubsection{Possibilistic conditioning}

The possibilistic conditioning consists in the revision of our initial knowledge, encoded by a possibility distribution $\Pi$, after the arrival of a new certain information $\phi \subseteq \Omega$. The initial distribution $\Pi$ is then replaced by another one, denoted $\Pi^{\prime}=\Pi(. \mid \phi)$. The two interpretations of the possibilistic scale (qualitative and quantitative) induce two definitions of possibilistic conditioning [4]. In this paper, we focus on min-based conditioning (qualitative one) defined by:

$$
\Pi(\omega \mid \phi)=\left\{\begin{array}{cc}
1 & \text { if } \pi(\omega)=\Pi(\phi) \text { and } \omega \vDash \phi i \\
\pi(\omega) & \text { if } \pi(\omega)<\Pi(\phi) \text { and } \omega \vDash \phi i \\
0 \text { otherwise }
\end{array}\right.
$$

\subsection{Min-based possibilistic network}

\subsubsection{Preliminaries}

There are two ways of knowledge representation: a logical representation and a graphical representation. In this paper we are interested to the graphical representation. It is qualitative network. A possibilistic network is an adaptation of the probabilistic (Bayesian) network, in the sense where we use the same graphic structure which is the direct acyclic graph (DAG)

A min-based possibilistic network [10] over a set of variables $\mathrm{V}$ denoted by $\Pi G_{\min }=$ $\left(G, \pi_{\min }\right)$ is characterized by:

- A graphical component: which is represented by a Directed Acyclic Graph (DAG) where nodes correspond to variables and arcs represent dependence relations between variables.

- Numerical components: these components quantify different links in the DAG by using local possibility distributions for each node $\mathrm{A}$ in the context of its parents denoted by $U_{A}$. More precisely:

- $\quad$ For every root node $A\left(U_{A}=\varnothing\right)$, uncertainty is represented by the a priori possibility degree $\pi(a)$, for each instance $\mathrm{a} \in \mathrm{D}_{\mathrm{A}}$, such that $\max \pi(a)=1$.

- $\quad$ For the rest of the nodes $A\left(U_{A} \neq \varnothing\right)$, uncertainty is represented by the conditional possibility degree $\pi\left(a \mid U_{A}\right)$, for each instance a $\in \mathrm{D}_{\mathrm{A}}$, and $U_{A} \in \mathrm{D}_{\mathrm{A}}$, such that $\max \pi\left(a \mid U_{A}\right)=1$, for any $U_{A}$.

The a priori and the conditional possibility degrees induce a unique joint possibility distribution defined by:

$$
\pi_{G}\left(A_{1}, \ldots, A_{n}\right)=\Pi_{\min }\left(A_{i} \mid U_{A i}\right)
$$




\subsubsection{Fusion of min-based possibilistic networks}

Merging uncertain information [6] is important to exploit complementarities between sources. It provides thus a global and complete point of view. In this paper, we are interested in conjunctive mode which makes sense if all sources are considered as equally and fully reliable. One of the basic conjunctive operators is the minimum operation (min). Given two min-based possibilistic networks $\Pi G_{\min }=\left(G, \pi_{G}\right)$ and $\Pi G^{\prime}{ }_{\min }=\left(G^{\prime}, \pi_{G^{\prime}}\right)$, the result of merging $\Pi G$ and $\Pi G^{\prime}$ is the possibilistic network $\Pi G_{\oplus}=\left(G_{\oplus}, \pi_{\oplus}\right)$ [15], such that:

$$
\forall \omega, \pi_{\oplus}(\omega)=\min \left(\pi_{G}(\omega), \pi_{G^{\prime}}(\omega)\right)
$$

The syntactic counterpart of the fusion of two possibility distributions, associated to two possibilistic networks, using the min operator is a new min-based possibilistic network whose definition depends on the union of the two initial ones. In [15], the authors propose two principal classes for merging min-based possibilistic networks:

- Fusion of two possibilistic networks $\Pi G$ and $\Pi G^{\prime}$ having the same network structure. The resulting network $\Pi G_{\oplus}$ retains the same structure: $G_{\oplus}=G=G^{\prime}$. The possibility degrees are computed as follows: for each variable $\mathrm{A}, \pi_{\oplus}\left(A \mid U_{A}\right)=\min \left(\pi_{G}\left(A \mid U_{A}\right), \pi_{G^{\prime}}\left(A \mid U_{A}\right)\right)$.

- Fusion of two possibilistic networks $\Pi G$ and $\Pi G^{\prime}$ with different structures. Two cases are distinguishable:

- The union of graphs is acyclic. In this case, the union of the two graphs is retained as the result of the fusion. The set of its variables is the union of the sets of variables belonging to $\Pi G$ and $\Pi G^{\prime}$. For each variable A, its parents are both $\Pi G$ and $\Pi G^{\prime}$.

- The union of graphs is cyclic. Further variables are added to eliminate cycles. The new conditional distributions of the new variables ensure equivalence between the new and the old variables.

For more details on the fusion of possibilistic networks see [15].

\section{QUALITATIVE POSSIBILISTIC DECISION}

In a problem of decision under uncertainty, knowledge of the decision-maker is generally not very informative. In other words, the agent does not know the real state of the world, but he knows only that this state belongs to a finite set of possible states. A decision system is defined by a finite set of states $S=\left\{s_{1}, s_{2}, \ldots, s_{n}\right\}$, a finite set of consequences $\mathrm{X}$, a set of decisions noted $D-=\left\{d_{1}, d_{2}, \ldots, d_{m}\right\}$, and a set of preferences among the consequences. Each decision $d_{i}: S \rightarrow X$ is a function that associates to every possible state of the world a consequence. The preferences among the consequences are encoded by the utility function $v: X \rightarrow U$ where $\mathrm{U}$ is a preferably ordinal scale.

The theory of possibility allows one to express the uncertain knowledge on different states of the world by using a possibility distribution. Indeed, it allows one to represent uncertain knowledge by distinguishing what is plausible to what is less plausible. It provides also a suitable mean to represent preferences on the consequences of decisions in order to distinguish the desirable consequences from the less desirable ones [9]. 
The uncertainty on the possible states of the world is represented by a normalized possibility distribution $\pi$ that associates to a set of state variables a value in the interval $[0,1]$. Likewise, the preferences of the agent are represented by a different possibility distribution $\mu$ that associates to a set of consequences a value in an ordinal scale $U$, represented by the interval [0,1] [8]. We assume that the uncertainties and preferences are commeasurable [5].

In the context of decision theory under uncertainty proposed by Savage, uncertainty of the agent is modeled by a probability distribution $\pi$ on the set of possible states of the world and its preferences by a utility function $\mu$ with real values on the set $\mathrm{X}$ of the possible consequences of his/her actions.

In contrast, in the possibilistic framework, knowledge of the agent is modeled by a normalized possibilistic distribution $\pi$ which is a function from states to a simply ordered scale $L$ of plausibility: for a world $\omega, \pi(\omega) \in L$ : represents the degree of likelihood that $\omega$ is the real state of the world. If we consider that the information possessed by the agent on the decision problem is purely ordinal, it is reasonable to think that not only his/her knowledge can be expressed by a possibilistic distribution but also his/her preferences [3][12]. A distribution of possibilities can be then seen as a utility [12] function.

Let $\mu$ be the possibility distribution representing the agent's preferences. $\mu$ takes its values in a simply orderly scale in $[0,1]$. As in Savage theory, an action is represented by a function $d$ that associates to a world an element of X [12]. The utility of an action (decision) $d$ in a state $\omega$ and whose consequence is $d(\omega) \in X$ can be evaluated by combining the possibility degrees $\pi(\omega)$ and the utilities $\mu(d(\omega))$ in an appropriate manner for all the possible states of world [12].

Two evaluation criteria have been proposed to achieve such combinations assuming some form of commensurability between the scales of plausibility and utility [2] [12]:

- Pessimistic criterion (Minimax). Criterion of a pessimistic decision maker: the chosen decision is that having the largest minimum utility :

$$
\mathrm{U}^{*}(\mathrm{~d})=\operatorname{minmax}_{\omega \in \Omega}\left(1-\pi_{\mathrm{Kd}}(\omega), \mu(\omega)\right)
$$

- Optimistic criterion (Maximin): Criterion of an optimistic decision maker: the chosen decision is that having the largest maximum utility :

$$
\mathrm{U}^{*}(\mathrm{~d})=\operatorname{maxmin}_{\omega \in \Omega}\left(\pi_{\mathrm{Kd}}(\omega), \mu(\omega)\right)
$$

In this work, we are interested in the optimistic criterion for the calculation of the decision.

Example 1: Let us consider the problem of deciding whether we should or not take an umbrella, knowing that it would rain. The two min-based possibilistic networks representing knowledge and preferences of the agent are denoted $\boldsymbol{\Pi} \boldsymbol{K}_{\min }$ and $\boldsymbol{\Pi} \boldsymbol{P}_{\min }$ respectively. Before presenting the possibilistic graphs, let us first present the set of nodes used in the networks and their meanings.

- R: It's raining.

- W: The grass is wet.

- UM: Take the umbrella.

- C: Cloudy atmosphere. 


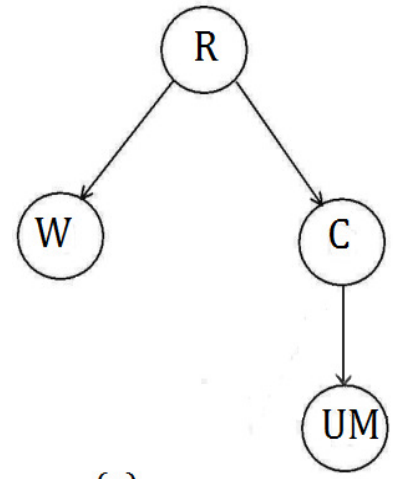

(a)

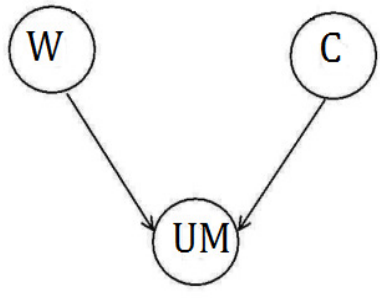

(b)

Figure1. The possibilistic networks of knowledge and preference of an agent

- Agent's knowledge: described by the min-based possibilistic network $\Pi K_{\min }=$ $\left(G_{K}, \pi_{K}\right)$, where the graphical component GK is given by Figure 1 (a). It contains one possible states of the world $\mathrm{R}$, one decision variable $\mathrm{UM}$ and two consequences $\{\mathrm{W}, \mathrm{C}\}$. The initial possibility distributions associated with $\Pi K_{\min }$ are given by Tables 1 and 2 . We suppose that the variables are binary.

\begin{tabular}{|ll|}
\hline $\mathrm{R}$ & $\pi_{\mathrm{K}}(\mathrm{R})$ \\
\hline $\mathrm{r} 1$ & 0.9 \\
$\mathrm{r} 2$ & 1.0 \\
\hline
\end{tabular}

Table 1 . Initial possibility distributions relative to $\Pi \mathrm{K}_{\mathrm{min}}$

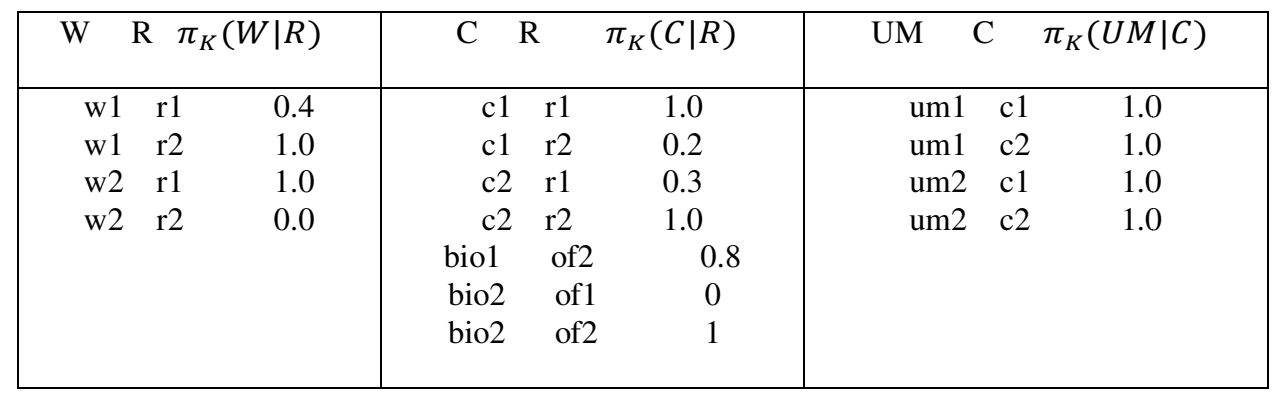

Table 2. Initial possibility distributions relative to $П \mathrm{~K}_{\mathrm{min}}$

- Agent's preferences: expressed by the min-based possibilistic network $\Pi P_{\min }=$ $\left(G_{P}, \mu\right)$, where the graphical component $G_{P}$, is given by Figure 1 (b). It contains one decision variable $U M$ and two consequences $\{\mathrm{W}, \mathrm{C}\}$. The initial possibility distributions associated with $\Pi P_{\min }$ are given by Tables 3 and 4 .

\begin{tabular}{|cc|cc|}
\hline $\mathrm{W}$ & $\mu(\mathrm{W})$ & $\mathrm{UM}$ & $\mu(\mathrm{UM})$ \\
\hline $\mathrm{w} 1$ & 1.0 & $\mathrm{um} 1$ & 1.0 \\
$\mathrm{w} 2$ & 1.0 & $\mathrm{um} 2$ & 1.0 \\
\hline
\end{tabular}

Table 3. Initial possibility distributions relative to $П \mathrm{P}_{\min }$ 


\begin{tabular}{|cccc|cccc|}
\hline UM & W & R & $\mu(\mathrm{UM} \mid \mathrm{W}$ R) & UM & W & R & $\mu(\mathrm{UM} \mid \mathrm{W}$ R) \\
\hline um1 & w1 & r1 & 1.0 & um2 & w1 & r1 & 1.0 \\
um1 & w1 & r2 & 1.0 & um2 & w1 & r2 & 0.0 \\
um1 & w2 & r1 & 1.0 & um2 & w2 & r1 & 0.8 \\
um1 & w2 & r2 & 1.0 & um2 & w2 & r2 & 1.0 \\
\hline
\end{tabular}

Table 4: Initial possibility distributions relative to $\Pi_{\min }$

\section{ON THE COMPUTATION OF OPTIMAL OPTIMISTIC DECISIONS BASED ON MIN-BASED FUSION}

This section presents the computation of qualitative possibilistic decision which is regarded as a problem of merging data from two possibility distributions: the first represents agent's beliefs and the second represents the qualitative utility. Knowledge and preferences of the agent are both represented by two separated min-based possibilistic networks, namely $\Pi K_{\min }=\left(G_{K}, \pi_{K}\right)$ and $\Pi P_{\min }=\left(G_{P}, \mu\right)$, respectively. In what follows, we propose a directed method for computing optimal optimistic decisions based on the fusion of $\pi_{K}$ and $\mu$ (or $\Pi K_{\min }$ and $\left.\Pi P_{\min }\right)$. Each decision d induces a possibility distribution $\pi_{K d}$ defined as follows:

$$
\pi_{\mathrm{k}_{\mathrm{d}}}(\omega)=\min \left(\pi_{\mathrm{k}}(\omega), \pi_{\mathrm{d}}(\omega)\right)
$$

We recall that making a decision comes down to choosing a subset $\mathrm{d}$ of the decision set $\mathrm{D}$ which maximizes the optimistic qualitative utility by:

$$
\mathrm{U}^{*}(\mathrm{~d})=\operatorname{maxmin}_{\omega \in \Omega}\left(\pi_{\mathrm{k}_{\mathrm{d}}}(\omega), \mu(\omega)\right)
$$

Where,

$$
\pi_{d}(\omega)=\left\{\begin{array}{cc}
1 & \text { if } \omega \vDash \phi i \\
0 & \text { otherwise }
\end{array}\right.
$$

Using equation (11), the optimistic utility decision $\mathrm{U}^{*}(\mathrm{~d})$ becomes:

$$
\mathrm{U}^{*}(\mathrm{~d})=\operatorname{maxmin}_{\omega \in \Omega}\left(\min \left(\pi_{\mathrm{k}_{\mathrm{d}}}(\omega), \mu(\omega)\right), \pi_{\mathrm{d}}(\omega)\right)
$$

Using technical merging of two min-based possibilistic networks, this Equation (12) down to:

$$
\mathrm{U}^{*}(\mathrm{~d})=\operatorname{maxmin}_{\omega \in \Omega}\left(\min \left(\pi_{\oplus}, \pi_{\mathrm{d}}(\omega)\right)\right.
$$

Example 2: The two DAGs $\left(G_{K}\right.$ and $\left.G_{P}\right)$ given in Example 1, Figure 1 have a different structures. Their union is acycles, the result of merging $\Pi K_{\min }$ and $\Pi P_{\min }$ is the min-based possibilistic network $\Pi G_{\oplus}=\left(G_{\oplus}, \pi_{\oplus}\right)$ where $\mathrm{G}_{\oplus}$, is given in Figure 2 . 


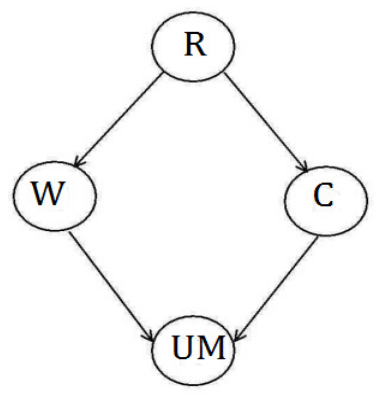

Figure2. The DAG $\mathrm{G}_{\oplus}$

The initial possibility distributions are given by Tables 5 and 6 .

\begin{tabular}{|cc|ccc|ccc|}
\hline $\mathrm{R}$ & $\pi_{\mathrm{K}}(\mathrm{R})$ & $\mathrm{W}$ & $\mathrm{R}$ & $\pi_{\mathrm{K}}(\mathrm{W} \mid \mathrm{R})$ & $\mathrm{C}$ & $\mathrm{R}$ & $\pi_{\mathrm{K}}(\mathrm{C} \mid \mathrm{R})$ \\
\hline $\mathrm{r} 1$ & 0.9 & w1 & $\mathrm{r} 1$ & 0.4 & $\mathrm{c} 1$ & $\mathrm{r} 1$ & 1.0 \\
$\mathrm{r} 2$ & 1.0 & w1 & $\mathrm{r} 2$ & 1.0 & $\mathrm{c} 1$ & $\mathrm{r} 2$ & 0.2 \\
& & w2 & $\mathrm{r} 1$ & 1.0 & $\mathrm{c} 2$ & $\mathrm{r} 1$ & 0.3 \\
& & w2 & $\mathrm{r} 2$ & 0.0 & $\mathrm{c} 2$ & $\mathrm{r} 2$ & 1.0 \\
& & & & & & & \\
\hline
\end{tabular}

Table 5. Initial possibility distributions relative to $\Pi G_{\oplus}$

\begin{tabular}{|cccc|cccc|}
\hline UM & W & R & $\mu(\mathrm{UM} \mid \mathrm{W} \mathrm{R})$ & $\mathrm{UM}$ & $\mathrm{W}$ & $\mathrm{R}$ & $\mu(\mathrm{UM} \mid \mathrm{W} \mathrm{R})$ \\
\hline um1 & w1 & $\mathrm{r} 1$ & 1.0 & um2 & w1 & $\mathrm{r} 1$ & 1.0 \\
um1 & w1 & r2 & 0.0 & um2 & w1 & $\mathrm{r} 2$ & 1.0 \\
um1 & w2 & r1 & 0.8 & um2 & w2 & r1 & 1.0 \\
um1 & w2 & r2 & 1.0 & um2 & w2 & r2 & 1.0 \\
& & & & bio2 & of1 & 0 \\
& & & & bio2 & of2 & 1 \\
\hline
\end{tabular}

Table 6. Initial possibility distributions relative to $\Pi G_{\oplus}$.

\subsection{Computing optimal decisions using moral graph}

Computing the optimistic optimal decisions amounts to find the normalization degree of the moral graph resulting from the merging of the two possibilistic networks codifying knowledge of the agent and its preferences respectively without going through the junction tree. Note that the construction of the moral graph is done only once and has a polynomial complexity. However, the stabilization procedure, multiple stabilization procedure and initialization (see below) (which are all three polynomials) are repeated for each decision $\mathrm{d}^{*}$.

\section{1) Building the moral graph.}

The construction of the possibilistic moral graph, noted $\mathcal{M} \mathcal{G}$, from the initial graph is done as follows:

- For each variable $\mathrm{Ai}$, form a cluster $\mathrm{C}_{i}=A_{i} \cup U_{A}$

- For each edge connecting two nodes $A_{i}$ and $A_{j}$ : form an undirected edge in the moral graph between the cluster $\mathrm{C}_{i}$ and the cluster $\mathrm{C}_{j}$ labeled with a separator $\mathrm{S}_{i j}$ corresponding to their intersection. 


\section{2) Initialization.}

For a given decision d, once the moral graph is built, we proceed to its quantification by taking into account the decision $d$ as follows:

- $\quad$ For each cluster $\mathrm{C}_{i}$, (resp. $\left.\mathrm{S}_{i j}\right) \pi_{\mathrm{Ci}}^{\mathrm{I}} \leftarrow 1$. (resp. $\mathrm{S}_{i j} \leftarrow 1$ )

- For each variable $A_{i}$, choose a cluster $\mathrm{C}_{i}$ containing $A_{i} \cup U_{A}$ $\pi_{C_{i}} \leftarrow \min \left(\pi_{C_{i}}, \pi_{\oplus}\left(A_{i} \mid U_{A}\right)\right)$.

- Encode the evidence $D=d_{i}$ as likelihood $\Lambda_{D}(d)$ :

$-$

$$
\Lambda_{D}(d): \begin{cases}1 & D \text { is instanciated as } d \\ 0 & D \text { is instanciated as a value } d^{\prime} \neq d\end{cases}
$$

- Identify a cluster $C_{i}$ containing D: $\pi_{\mathrm{Ci}}^{\mathrm{I}} \leftarrow \min \left(\pi_{\mathrm{Ci}}^{\mathrm{I}}, \Lambda_{D}\right)$.

Proposition 1: Let $\Pi K_{\min }=\left(G_{K}, \pi_{K}\right)$, be a min-based possibilistic network representing agent's beliefs and $\Pi P_{\min }=\left(G_{P}, \mu\right)$ be a min-based possibilistic network representing agent's preferences. Let $\Pi G_{\oplus}=\left(G_{\oplus}, \pi_{\oplus}\right)$ be the result of merging $\Pi K_{\min }$ and $\Pi P_{\min }$ using the min operator. Let $\mathcal{M G}$, be the moral graph corresponding to $\Pi G_{\oplus}$ generated using the above initialization procedure. Then,

$$
\mathrm{U}^{*}(\mathrm{~d})=\max _{\omega \in \Omega}(\mathcal{M} \mathcal{G}(\omega))
$$

Where $U^{*}(d)$ is given in Equation 13 .

\section{3) Simple Stability Procedure.}

The simple stabilization procedure ensures that the potential of each clique is in agreement with that of its neighbors. This procedure is applied through a mechanism of passage of messages between different cliques. Indeed, each separator collects information from its corresponding cliques in order to distribute it then to each of them in order to update them.

The potentials of any adjacent clusters $\mathrm{C}_{i}$ and $\mathrm{C}_{j}$ (with separator $\mathrm{S}_{i j}$ ) are updated as follows:

- Collect evidence (Update separator) :

$$
S_{i j}^{t+1} \leftarrow \min \left(\max C \mathrm{Ci} / \mathrm{Sij}_{\mathrm{Ci}}, \max _{\mathrm{Cj} / \mathrm{Sij}} \pi_{\mathrm{Ci}}^{\mathrm{t}}\right)
$$

- Distribute evidence (Update clusters) :

$$
\begin{aligned}
\pi_{C i}^{t+1} & \leftarrow \min \left(\pi_{\mathrm{Ci}}^{t}, \pi_{\mathrm{Sij}}^{t+1}\right) \\
\pi_{C j}^{t+1} & \leftarrow \min \left(\pi_{\mathrm{Cj}}^{t}, \pi_{\mathrm{Sij}}^{t+1}\right)
\end{aligned}
$$

This procedure is defined as follows:

Definition 1: Let $C_{i}$ and $C_{j}$ be two adjacent clusters in a moral graph $\mathcal{M} \mathcal{G}$, and let $S_{i j}$ be their separator. The separator $S_{i j}$ is said to be stable if:

$$
\max \operatorname{Ci}_{\mathrm{S} i j} \pi_{\mathrm{Ci}}^{\mathrm{I}}=\max _{\mathrm{Cj} / \mathrm{Sij}} \pi_{\mathrm{Ci}}^{\mathrm{I}}
$$


Where $\max { }_{\mathrm{Ci} / \mathrm{Sij}} \pi_{\mathrm{C}_{\mathrm{i}}}$ is the marginal distribution of $\mathrm{S}_{i j}$ defined from $\pi_{\mathrm{Ci}}^{\mathrm{I}}$ (resp. $\pi_{\mathrm{Cj}}^{\mathrm{I}}$ ). A moral graph $\mathcal{M} \mathcal{G}$ is stable if all its separators are stable.

Proposition 2: Let $\mathcal{M G}$ be a stabilized moral graph, let $\pi_{\mathcal{M} \mathcal{G}}$ be the joint distribution encoded by MG after the initialization procedure. Then,

$$
\forall C_{i}, \max \pi_{\mathcal{M G}}^{S} \geq \alpha
$$

Where, $\alpha$ is the maximum value in all clusters.

\section{4) Multiple Stability Procedure.}

[1] Proved that the simple stabilization procedure does not always guarantee accurate marginal. One needs to stabilize each clique with respect to all of its adjacent cliques but this can turns out to be very costly in terms of calculation if the number of cliques is important. For that, [1] has proposed to follows several steps in stabilizing the possibilistic moral graph over subsets of its adjacent cliques. Authors of [1] have proposed several progressive stabilization procedures based on $\mathrm{n}$ parents, $\mathrm{n}$ children, $\mathrm{n}$ parents children and $\mathrm{n}$ neighbors by varying the value of $\mathrm{n}$ from 2 up to the cardinality of the considered subset. To illustrate the multiple stabilization procedure, we consider the case of two parent's stabilization. The principle of this procedure is to ensure for each clique, with at least two parents, its stabilization over each pair of parents. Once stability has been reached, the calculation of qualitative utility over a decision $\mathrm{d}$ will be obtained as follows:

Proposition 3. Let $\Pi K_{\min }=\left(G_{K}, \pi_{K}\right)$ be a min-based possibilistic network representing agent's beliefs and $\Pi P_{\min }=\left(G_{P}, \mu\right)$ a min-based possibilistic network representing agent's preferences. $\Pi G_{\oplus}$ is the result of merging of $\Pi K_{\min }$ and $\Pi P_{\min }$ by using the min operator. Let $\mathcal{M} \mathcal{G}$ be the moral graph of $\Pi G_{\oplus}$. The computation of optimistic decisions returns to calculate the normalization degree of MG:

$$
\mathrm{U}^{*}(\mathrm{~d})=\max _{C_{i}}\left(\pi_{C_{i}}\right)
$$

\subsection{Algorithm}

The computation of the optimal optimistic decisions is obtained using the following algorithm.

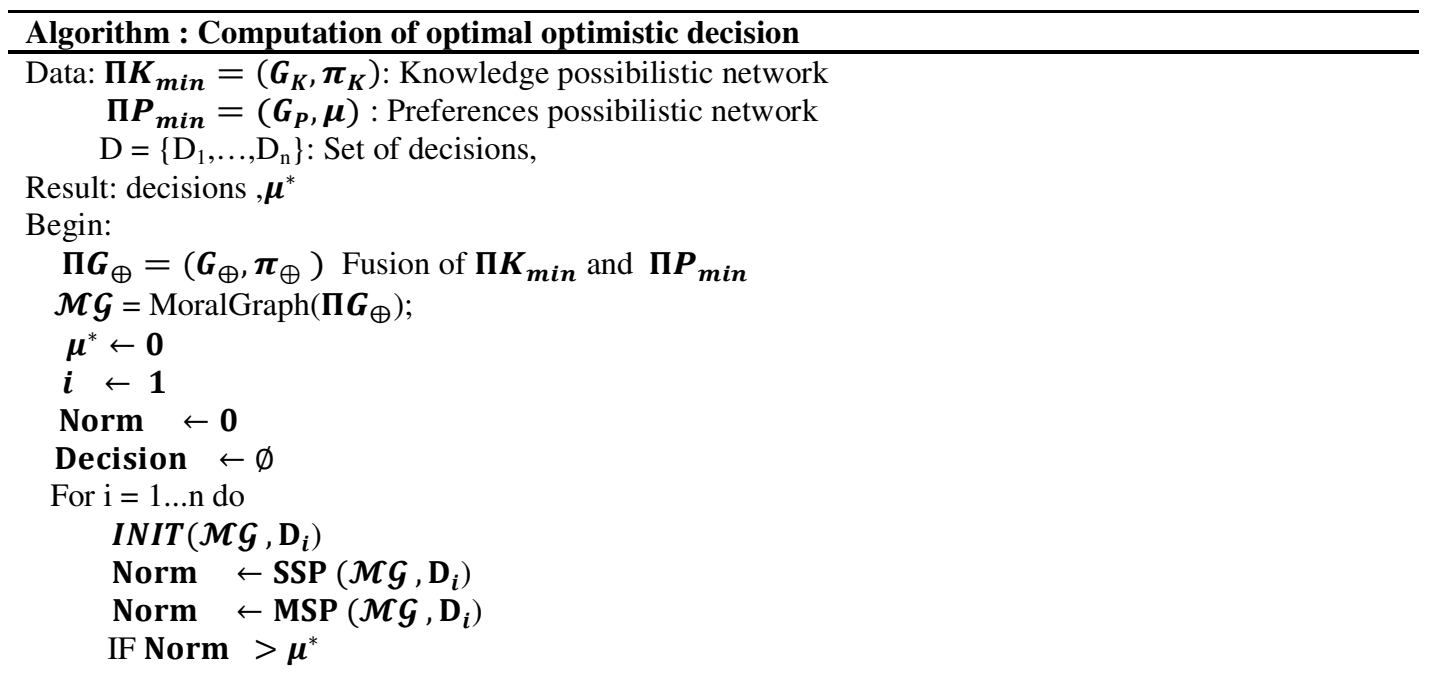




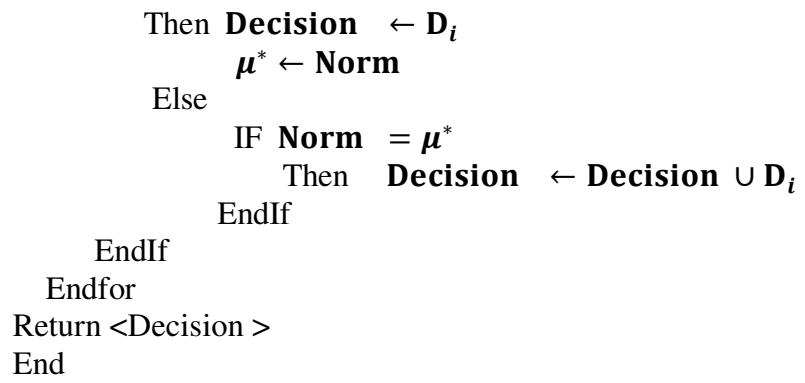

Example 3: Let us continue with Example 2. We need to compute the optimal optimistic decision $\mathrm{UM}=\{$ um1; um2 $\}$. First, we start by constructing the moral graph (see Figure3) associated with the graph $G_{\oplus}$ (Figure 2) representing the fusion of $\Pi K_{\min }$ and $\Pi P_{\min }$. The resulted moral graph contains four cluster $\mathrm{C} 1=\{\mathrm{R}\}, \mathrm{C} 2=\{\mathrm{R}, \mathrm{W}\}, \mathrm{C} 3=\{\mathrm{R}, \mathrm{C}\}$ and $\mathrm{C} 4=\{\mathrm{R}, \mathrm{W}, \mathrm{UM}\}$ and their separator $S 12=\{R\}, S 12=\{R\}, S 13=\{R\}, S 23=\{R\}, S 24=\{w\}$ and $S 34=\{c\}$.

Then, for each decision value in $\mathrm{UM}=\{\mathrm{um} 1 ; \mathrm{um} 2\}$, we must run the algorithm in order to compute the normalization degree associated with the moral graph.

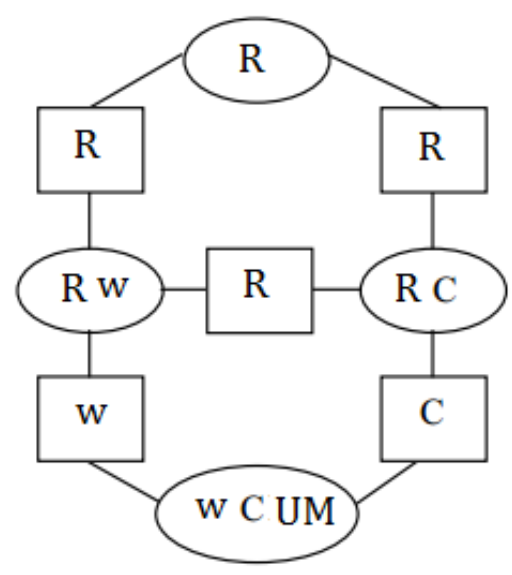

Figure.3. Moral Graph MG of th DAG in Figure 2

\begin{tabular}{|ll|ll|}
\hline \multicolumn{2}{|c|}{$\pi_{\mathcal{M G}}$} & $\omega$ & $\pi_{\mathcal{M G}}$ \\
\hline r1 w1 c1 um1 & 0.3 & r2 w1 c1 um1 & 0.0 \\
r1 w1 c1 um2 & 0.3 & r2 w1 c1 um2 & 0.0 \\
r1 w1 c2 um1 & 0.0 & r2 w1 c2 um1 & 0.0 \\
r1 w1 c2 um2 & 1.0 & r2 w1 c2 um2 & 0.0 \\
r1 w2 c1 um1 & 0.3 & r2 w2 c1 um1 & 0.8 \\
r1 w2 c1 um2 & 0.3 & r2 w2 c1 um2 & 0.9 \\
r1 w2 c2 um1 & 0.4 & r2 w2 c2 um1 & 0.2 \\
r1 w2 c2 um2 & 0.4 & r2 w2 c2 um2 & 0.2 \\
\hline
\end{tabular}

Table 7. Joint distribution $\pi_{\mathcal{M G}}$

\section{Step 1: UM= um1}

In this case, the fact that $\mathrm{UM}=\mathrm{um} 1$ is encoded interns of likelihood a s follows: 


$$
\Lambda_{U M}(u m 1): \begin{cases}1 & U M \text { is instanciated as um } 1 \\ 0 & U M \text { is instanciated as um } 2\end{cases}
$$

The table 8 represents the joint distribution encoded by $\mathcal{M G}$ after the initialization procedure us.

\begin{tabular}{|ll|ll|}
\hline \multicolumn{2}{|c|}{$\pi_{\mathcal{M C} \mathcal{G}}$} & $\omega$ & $\pi_{\mathcal{M G}}$ \\
\hline r1 w1 c1 um1 & 0.3 & r2 w1 c1 um1 & 0.0 \\
r1 w1 c1 um2 & 0.0 & r2 w1 c1 um2 & 0.0 \\
r1 w1 c2 um1 & 0.0 & r2 w1 c2 um1 & 0.0 \\
r1 w1 c2 um2 & 0.0 & r2 w1 c2 um2 & 0.0 \\
r1 w2 c1 um1 & 0.3 & r2 w2 c1 um1 & 0.8 \\
r1 w2 c1 um2 & 0.0 & r2 w2 c1 um2 & 0.0 \\
r1 w2 c2 um1 & 0.4 & r2 w2 c2 um1 & 0.2 \\
r1 w2 c2 um2 & 0.0 & r2 w2 c2 um2 & 0.0 \\
\hline
\end{tabular}

Table 8 . Joint distributions $\pi_{\mathcal{M G}}$ after the initialization procedure

Once the moral graph is quantified, then the simple stabilization procedure allows us to compute the normalization degree of the moral graph which corresponds to the normalization degree of any cluster. Using this procedure, we obtain:

\begin{tabular}{|c|c|c|c|c|c|c|c|c|c|}
\hline $\mathrm{R}$ & $\pi_{\mathrm{C} 1}$ & $\mathrm{R} \quad \mathrm{W}$ & $\pi_{\mathrm{C} 2}$ & $\mathrm{R} \quad \mathrm{C}$ & $\pi_{\mathrm{C} 3}$ & W C UM & $\pi_{\mathrm{C} 4}$ & W C UM & $\pi_{\mathrm{C} 4}$ \\
\hline $\begin{array}{l}\mathrm{r} 1 \\
\mathrm{r} 2\end{array}$ & $\begin{array}{l}0.9 \\
0.9\end{array}$ & $\begin{array}{ll}\mathrm{r} 1 & \mathrm{w} 1 \\
\mathrm{r} 1 & \mathrm{w} 2 \\
\mathrm{r} 2 & \mathrm{w} 1 \\
\mathrm{r} 2 & \mathrm{w} 2\end{array}$ & $\begin{array}{l}0.4 \\
0.9 \\
0.9 \\
0.0\end{array}$ & $\begin{array}{ll}\mathrm{r} 1 & \mathrm{w} 1 \\
\mathrm{r} 1 & \mathrm{w} 2 \\
\mathrm{r} 2 & \mathrm{w} 1 \\
\mathrm{r} 2 & \mathrm{w} 2\end{array}$ & $\begin{array}{l}0.9 \\
0.3 \\
0.2 \\
0.9\end{array}$ & $\begin{array}{l}\text { w1 c1 um1 } \\
\text { w1 c1 um2 } \\
\text { w1 c2 } 1 \text { um1 } 1 \\
\text { w1 c2 um2 }\end{array}$ & $\begin{array}{l}0.9 \\
0.0 \\
0.0 \\
0.0\end{array}$ & $\begin{array}{l}\text { w2 } \\
\text { c1 } 1 \text { um1 } \\
\text { w2 } c 1 \text { um2 } \\
\text { w2 c2 um1 } \\
\text { w2 c2 um2 }\end{array}$ & $\begin{array}{l}0.8 \\
0.0 \\
0.9 \\
0.0\end{array}$ \\
\hline
\end{tabular}

Table 9. Normalized potentials with UM=um1

$\max _{\pi C 1}=\max _{\pi C 2}=\max _{\pi C 3}=\max _{\pi C 4}=0.9$

From the table 8 we can check that: $\mathrm{h}\left(\pi_{\mathcal{M G}}\right)=0.8 \neq 0.9$, which means that the moral graph is not consistent. So we must to re-stabilize the moral graph using the multiple. stability procedure. Using this procedure, we obtain:

$\max _{\pi C 1}=\max _{\pi C 2}=\max _{\pi C 3}=\max _{\pi C 4}=0.8$

The normalization degree of the moral graph is: $\mathrm{U}^{*}(\mathrm{um} 1)=0.8$

\section{Step 2: UM= um2}

We repeat the same procedure described in the previous step, with:

Then, we get:

$$
\Lambda_{U M}(u m 2): \begin{cases}1 & U M \text { is instanciated as um } 2 \\ 0 & U M \text { is instanciated as um } 1\end{cases}
$$

$\mathrm{U}^{*}(\mathrm{um} 2)=\max _{\pi C 1}=\max _{\pi C 2}=\max _{\pi C 3}=\max _{\pi C 4}=1.0$ 
Thus, we can conclude that the optimal decision is $\mathbf{U M}=\mathbf{u m} \mathbf{2}$ with the maximal qualitative utility which equals 1.0

\section{CONCLUSION}

In this paper, we proposed a new approximate approach for the computation of the qualitative possibilistic optimal optimistic decision in a graphical context. Our approach first merges possibilistic networks associated with uncertain knowledge and possibilistic networks associated with agent's preferences. We then showed that computing optimistic decisions comes down to computing a normalization degree of the moral graph associated to the result graph of merging agent's beliefs and preferences networks.

This approach allows one to avoid the transformation of the initial graph into a junction tree which is known as a difficult problem. This approach is interesting when accurate approaches fail, i.e., when the generation of the local possibility distributions by the standard algorithm is impossible or takes a too long response time. In such case, our approach provides answers that are very close to the exact marginal distributions.

\section{REFERENCES}

[1] N. Ben Amour. Qualitative Possibilistic Graphical models From Independence to propagation algorithm. PhD thesis, Université d'Artois, 2002.

[2] D. Dubois, J. Lang, and H. Prade. Possibilistic logic. In Handbook of Logic in Articial Intelligence and Logic Programming, (D. Gabbay et al., eds, 3, Oxford University Press :pages 439-513, 1994.

[3] D. Dubois, D. Le Berre, H. Prade, and R. Sabaddin. Using possibilistic logic for modeling qualitative decision: Atms based algorithms. In Fundamenta Informaticae, 37 :1-30, 1999.

[4] D. Dubois and H. Prade. (with the collaboration of H. Farreny, R. Martin-Clouaire and C. Testemale). Possibility Theory - An Approach to Computerized Processing of Uncertainty. Plenum Press, New York., 1988

[5] D. Dubois and H. Prade. Possibility theory and data fusion in poorly informed environments. In Control Engineering Practice, volume 2(5), pages 811823, 1994.

[6] D. Dubois and H. Prade. Possibility theory as a basis for qualitative decision theory. In 14th International Joint Conference on Artificial Intelligence (IJCAI'95), Montréal, pages 1924-1930, 1995.

[7] D. Dubois and H. Prade. Possibility theory: qualitative and quantitative aspects. In Handbook of Defeasible Reasoning and Uncertainty Management Systems. (D. Gabbay, Ph. Smets, eds.), Vol. 1: Quantified Representations of Uncertainty and Imprecision, (Ph. Smets, ed.) Kluwer, Dordrecht: 169226, 1998.

[8] F. Haned-Khellaf S. Benferhat and I. Zeddigha. Computing optimal optimistic decisions using minbased possibilistic networks. In North American Fuzzy Information Processing Society, Berkeley NAFIPS 2012, JUIN 2012.

[9] L. Garcia and R. Sabbadin. Diagrammes d'influence possibilistes. Revue d'Intelligence Artificielle, 21(4): 521-554, 2007.

[10] J. Gebhardt and R. Kruse. Background and perspectives of possibilistic graphical models. In 4th European Conference on Symbolic and Quantitative Approaches to Reasoning and Uncertainty (ECSQARU'97), LNAI 2143, pages 108-121, 1997.

[11] A. Mokhtari S. Benferhat, F. Haned-Khellaf and I. Zeddigha. A possibilistic graphical model for handling decision problems under uncertainty. In 8th conference of the European Society for Fuzzy Logic and Technology, EUSFLAT-2013, September 2013.

[12] R. Sabbadin. Une approche logique de la résolution de problèmes de décision sous incertitude basée sur les atms. In Actes du 11ème Congrés Reconnaissance des Formes et Intelligence Artificielle (RFIA'98), Clermont-Ferrand, pages 391- 400, 20-22 janvier 1998.

[13] P.P Shenoy. Valuation based systems: A framework for managing uncertainty in expert systems. In Fuzzy Logic for the Management of Uncertainty, pages 83104. L. A. Zadeh and J. Kacprzyk, Eds. John Wiley and Sons, New York, NY, 1992. 
[14] P.P Shenoy. A comparison of graphical techniques for decision analysis. In European Journal of Operational Research, volume 78, pages 1-21, 1994.

[15] F.Titouna. Fusion de réseaux causaux possibilistes. PhD thesis, Université d'Artois, 2009.

[16] L. Zadeh. Fuzzy sets as a basis for a theory of possibility. Fuzzy Sets and Systems, 1 :3-28, 1978. 